\title{
NONSMOOTH DATA ERROR ESTIMATES FOR APPROXIMATIONS OF AN EVOLUTION EQUATION WITH A POSITIVE-TYPE MEMORY TERM
}

\author{
CH. LUBICH, I.H. SLOAN, AND V. THOMÉE
}

\begin{abstract}
We study the numerical approximation of an integro-differential equation which is intermediate between the heat and wave equations. The proposed discretization uses convolution quadrature based on the first- and second-order backward difference methods in time, and piecewise linear finite elements in space. Optimal-order error bounds in terms of the initial data and the inhomogeneity are shown for positive times, without assumptions of spatial regularity of the data.
\end{abstract}

\section{INTRODUCTION}

We shall consider initial value problems of the form

$$
\begin{aligned}
u_{t}+\int_{0}^{t} \beta(t-s) A u(s) d s & =f(t) \quad \text { for } t>0, \\
u(0) & =u_{0} .
\end{aligned}
$$

Here, $u_{t}=\partial u / \partial t$ and $A$ is a selfadjoint positive definite second-order elliptic partial differential operator in $\Omega \subset R^{d}$, with Dirichlet boundary conditions, or, more generally, a positive definite linear operator in a real Hilbert space $H$. The kernel $\beta$ is assumed to be real-valued and positive definite, i.e., for each $T>0$ the kernel $\beta$ belongs to $L_{1}(0, T)$ and satisfies

$$
\int_{0}^{T} \varphi(t) \int_{0}^{t} \beta(t-s) \varphi(s) d s d t \geq 0 \quad \text { for all } \varphi \in C[0, T] .
$$

As is easily seen by an energy argument, the positive definiteness of $\beta$ and $A$ implies stability for the solution of (1.1), or

$$
\|u(t)\| \leq\left\|u_{0}\right\|+2 \int_{0}^{t}\|f(s)\| d s \text { for } t>0
$$

where $\|\cdot\|$ denotes the norm in the Hilbert space $H$.

Such problems, or nonlinear versions thereof, are used to model viscoelasticity and heat conduction in materials with memory, cf. the references in [3]. When $\beta$ is

Received by the editor August 30, 1994.

1991 Mathematics Subject Classification. Primary 45K05, 65M60, 65D32.

Key words and phrases. Evolution equation, memory term, nonsmooth data, convolution quadrature. 
smooth such equations are hyperbolic in nature, whereas if $\beta$ has a weak singularity at $t=0$, they exhibit certain features of parabolic equations. In particular, when

$$
\beta(t)=t^{\alpha-1} / \Gamma(\alpha), \quad 0<\alpha<1,
$$

which is the case we shall consider in this paper, the homogeneous equation has a smoothing property corresponding to the inequality

$$
\left\|u^{(m)}(t)\right\|_{\mu+\nu} \leq C t^{-(\alpha+1) \mu-m}\left\|u_{0}\right\|_{\nu} \text { for } t>0 \text {, where }\left\|u_{0}\right\|_{\nu}=\left\|A^{\nu / 2} u_{0}\right\|,
$$

for $\nu \geq 0$, for $|\mu| \leq 1$ if $m>0$, and for $0 \leq \mu \leq 1$ if $m=0$, but with no further smoothing in the spatial variables. Here, $C$ depends on $\alpha$ and $m$.

The numerical solution of the problem (1.1) was studied in, e.g., [1, 3, 4, 6]. The methods considered in [3] and [4] were based on the standard finite element method in the spatial variable, together with some basic time-stepping methods, with appropriate quadrature formulas applied to the integral term. The analysis in [3] uses a combination of energy arguments and Fourier analysis in the time variable, and requires constant time steps. In [4] extensions to variable time steps were studied using only energy methods. The results in [3] and [4] show optimalorder error bounds which hold uniformly for small $t$, under specified smoothness assumptions. In [4] such uniform error bounds were derived also for solutions with weak singularities at $t=0$, when certain estimates of type (1.5) hold, provided the time levels are suitably graded near $t=0$. Our present main purpose is to show that in the case of constant time steps, the error is of optimal order for the homogeneous equation, when $t$ is bounded away from 0 , even without further assumptions than $u_{0} \in H$, provided the memory term is appropriately approximated. (Similar results are well known for parabolic equations, cf., e.g., [7].) We also show some related results for the inhomogeneous equation.

Our analysis will use Laplace transforms. The assumption that $A$ is positive definite implies that $A$ generates an analytic semigroup, so that for any $\omega<\pi$ and with $M=M_{\omega}$, we have the resolvent estimate

$$
\left\|(z I+A)^{-1}\right\| \leq \frac{M}{|z|} \quad \text { for } \quad z \in \Sigma_{\omega}=\{z \neq 0:|\arg z|<\omega\} .
$$

This estimate, with $\omega>\pi / 2$, will be used in an essential way below. In fact, we could have replaced our assumption that $A$ is positive definite by the property (1.6), with $(1+\alpha) \pi / 2<\omega<\pi$, where $\alpha$ is the parameter in (1.4), thus allowing more general nonsymmetric elliptic operators $A$. In the case of discretization in time our analysis also generalizes to a Banach space framework.

We consider first the case of discretization in space only, and let $S_{h}$ denote the piecewise linear functions on a triangulation of $\Omega$ of standard type so that

$$
\inf _{\chi \in S_{h}}\left\{\|u-\chi\|+h\|u-\chi\|_{1}\right\} \leq C h^{2}\|u\|_{2},
$$

where $\|\cdot\|$ now denotes the norm in $L_{2}(\Omega)$. (Higher-order elements could be similarly considered.) With $A(\cdot, \cdot)$ denoting the bilinear form associated with $A$, and $(\cdot, \cdot)$ the inner product in $L_{2}(\Omega)$, the spatially discrete problem is then to find $u_{h}(t) \in S_{h}$ for $t \geq 0$ such that

$$
\begin{aligned}
\left(u_{h, t}, \chi\right)+\int_{0}^{t} \beta(t-s) A\left(u_{h}(s), \chi\right) d s & =(f, \chi) \quad \text { for } \chi \in S_{h}, t>0, \\
u_{h}(0) & =u_{0 h} \approx u_{0} .
\end{aligned}
$$


For this problem it was shown in [3] that, with $u_{0 h}$ appropriately chosen,

$$
\left\|u_{h}(t)-u(t)\right\| \leq C h^{2}\left\{\left\|u_{0}\right\|_{2}+\int_{0}^{t}\left\|u_{t}\right\|_{2} d s\right\} \quad \text { for } t>0 .
$$

Our purpose being to reduce the regularity assumptions on the solution, or data, we shall show below that if $u_{0 h}=P_{h} u_{0}$, where $P_{h}$ is the $L_{2}$-projection onto $S_{h}$, then, for $t>0$, with $C=C_{\alpha}$,

$$
\left\|u_{h}(t)-u(t)\right\| \leq C h^{2}\left\{t^{-\alpha-1}\left\|u_{0}\right\|+t^{-\alpha}\|f(0)\|+\int_{0}^{t}(t-s)^{-\alpha}\left\|f_{t}(s)\right\| d s\right\} .
$$

We now turn to discretization in time only, and assume for the time being that $A$ is a positive definite operator in a Hilbert space $H$. Let $k$ be the time step, $t_{n}=n k$, and $U^{n}$ the approximation of $u\left(t_{n}\right)$. We shall first consider the backward Euler method, and thus use $\bar{\partial} U^{n}:=\left(U^{n}-U^{n-1}\right) / k$ to approximate the time derivative in (1.1). For the integral we apply a quadrature formula

$$
q_{n}(\varphi)=\sum_{j=0}^{n} \omega_{n j} \varphi^{j} \approx \int_{0}^{t_{n}} \beta\left(t_{n}-s\right) \varphi(s) d s, \quad \text { where } \varphi^{j}:=\varphi\left(t_{j}\right),
$$

and thus consider the time discrete problem

$$
\begin{aligned}
\bar{\partial} U^{n}+q_{n}(A U) & =f^{n}:=f\left(t_{n}\right) \quad \text { for } n \geq 1, \\
U^{0} & =u_{0} .
\end{aligned}
$$

We will consider quadrature formulas $q_{n}(\varphi)$ which are positive definite in the sense

$$
Q_{N}(\varphi):=k \sum_{n=1}^{N} q_{n}(\varphi) \varphi^{n} \geq 0, \quad \forall \varphi, N,
$$

which we may think of as a discrete analogue of (1.2). In [3] it is shown that this provides a sufficient condition for stability, and that

$$
\left\|U^{N}\right\| \leq\left\|u_{0}\right\|+2 k \sum_{n=1}^{N}\left\|f^{n}\right\|,
$$

which is a discrete analogue of (1.3). With local and global quadrature errors defined by

$$
\varepsilon_{n}(\varphi):=q_{n}(\varphi)-\int_{0}^{t_{n}} \beta\left(t_{n}-s\right) \varphi(s) d s \quad \text { and } \quad \mathcal{E}_{N}(\varphi):=k \sum_{n=1}^{N}\left\|\varepsilon_{n}(\varphi)\right\|,
$$

respectively, the stability yields the preliminary error estimate (see [3])

$$
\left\|U^{n}-u\left(t_{n}\right)\right\| \leq C k \int_{0}^{t_{n}}\left\|u_{t t}\right\| d t+2 \mathcal{E}_{n}(A u) .
$$

In particular, if $\varepsilon_{n}(\varphi)$ is of first-order accuracy and $u$ smooth, this error bound is $O(k)$. In the case that $A$ is a second-order elliptic operator and $U^{n}$ is the time discretization of the spatially semidiscrete equation (1.7) with the appropriate $U^{0} \approx u_{0}$, then the term on the right in (1.8) has to be added to the error bound (1.13) to obtain a complete error bound.

In [3], and previously in [5], various quadrature rules satisfying the stability criterion (1.11) were proposed. In particular, for $\beta \in L_{1}(0, T)$ and positive definite 
it was shown that with $\bar{\varphi}$ the piecewise constant function taking the value $\varphi^{j}$ in $\left(t_{j-1}, t_{j}\right]$, the quadrature rule defined by

$$
q_{n}(\varphi)=\int_{0}^{t_{n}} \beta\left(t_{n}-s\right) \bar{\varphi}(s) d s=\sum_{j=1}^{n} \kappa_{n-j} \varphi^{j}, \quad \text { i.e., with } \kappa_{j}=\int_{t_{j}}^{t_{j+1}} \beta(s) d s,
$$

is positive definite, and that

$$
\mathcal{E}_{n}(\varphi) \leq C_{T} k \int_{0}^{t_{n}}\left\|\varphi_{t}\right\| d s .
$$

Using this error estimate together with a smoothing property of type (1.5), we can easily show, in the case of the homogeneous equation and discretization in time only, that

$$
\left\|U^{n}-u\left(t_{n}\right)\right\| \leq C k \int_{0}^{t_{n}}\left(\left\|u_{t t}\right\|+\left\|A u_{t}\right\|\right) d t \leq C_{\varepsilon} k\left\|u_{0}\right\|_{2+\varepsilon} \quad \text { for } \varepsilon>0 .
$$

As in (1.9), however, we want to assume only $u_{0} \in H$. For $\beta$ as in (1.4) and for $q_{n}(\varphi)$ chosen in a specific way, we shall be able to show that, with $C=C_{\alpha}$,

$$
\left\|U^{n}-u\left(t_{n}\right)\right\| \leq C k t_{n}^{-1}\left\|u_{0}\right\| \text { for } n \geq 1,
$$

in the homogeneous case, together with an associated uniform in time first-order estimate for the inhomogeneous equation with vanishing initial data. This will be achieved by using "convolution quadrature" as introduced by Lubich [2], or, equivalently, by thinking of $\beta * \varphi$ as the fractional integral $(\partial / \partial t)^{-\alpha} \varphi$, and then using the same formal approximation of $\partial / \partial t$ in both terms on the left in (1.1). Corresponding to the backward Euler approximation of $\partial / \partial t$, which has the characteristic polynomial $(1-\zeta) / k$, one thus chooses the quadrature coefficients to have the generating function $\hat{\beta}((1-\zeta) / k)$, where $\hat{\beta}(z)$ is the Laplace transform of $\beta(t)$, i.e., $\hat{\beta}(z)=z^{-\alpha}$ for $\beta$ in (1.4). In other words, one considers the quadrature formula

$$
q_{n}^{0}(\varphi)=k^{\alpha} \sum_{j=0}^{n} \beta_{n-j} \varphi^{j}, \quad \text { where } \sum_{j=0}^{\infty} \beta_{j} \zeta^{j}=(1-\zeta)^{-\alpha}, \beta_{j}=(-1)^{j}\left(\begin{array}{c}
-\alpha \\
j
\end{array}\right) .
$$

It will prove convenient, however, to slightly modify this formula by omitting the term with $j=0$ in (1.15), so that $q_{n}(\varphi)=k^{\alpha} \sum_{j=1}^{n} \beta_{n-j} \varphi^{j}$. We shall show that the latter quadrature formula is positive definite, which implies, in particular, that the smooth data estimates of [3] hold. (For the special case $\alpha=1 / 2$ such a method was discussed in [6], cf. also [1].) In the case of a second-order elliptic $A$, with $U^{n}$ the completely discrete solution and with $u_{0 h}=P_{h} u_{0}$ and $f=0$, we have, by combination of (1.9) and (1.14), with $C=C_{\alpha}$,

$$
\left\|U^{n}-u\left(t_{n}\right)\right\| \leq\left\|U^{n}-u_{h}\left(t_{n}\right)\right\|+\left\|u_{h}\left(t_{n}\right)-u\left(t_{n}\right)\right\| \leq C\left(k t_{n}^{-1}+h^{2} t_{n}^{-\alpha-1}\right)\left\|u_{0}\right\|,
$$

showing optimal-order convergence in both space and time for $t_{n}>0$, with no regularity assumption other than $u_{0} \in L_{2}(\Omega)$.

In order to achieve higher accuracy, we next consider a time-stepping scheme for (1.1) which is based on the second-order backward difference approximation to $u_{t}$ defined by

$$
\bar{D} U^{n}:=\left(\frac{3}{2} U^{n}-2 U^{n-1}+\frac{1}{2} U^{n-2}\right) / k \text { for } n \geq 2 .
$$


We note that the characteristic polynomial associated with this operator is $\delta(\zeta) / k$, where $\delta(\zeta)=\frac{3}{2}-2 \zeta+\frac{1}{2} \zeta^{2}$. The above then suggests that we use for the approximation of the integral a quadrature rule of the form $q_{n}^{0}(\varphi)=k^{\alpha} \sum_{j=0}^{n} \beta_{n-j} \varphi^{j}$, where now the $\beta_{j}$ have the generating function $k^{\alpha} \sum_{j=0}^{\infty} \beta_{j} \zeta^{j}=\hat{\beta}(\delta(\zeta) / k)$, i.e., for the kernel (1.4),

$$
\sum_{j=0}^{\infty} \beta_{j} \zeta^{j}=\delta(\zeta)^{-\alpha}, \quad \text { with } \delta(\zeta)=\frac{3}{2}-2 \zeta+\frac{1}{2} \zeta^{2} .
$$

It is known from [2] that this formula is of second order when applied to smooth functions with $\varphi(0)=0$, but is less accurate when $\varphi(0) \neq 0$, e.g., when $\varphi(t) \equiv 1$. We shall therefore again use a modification, which this time may be written $q_{n}(\varphi)=$ $k^{\alpha}\left(\sum_{j=1}^{n} \beta_{n-j} \varphi^{j}+\frac{1}{2} \beta_{n-1} \varphi^{0}\right)$. With this choice, we thus define our scheme to be

$$
\begin{aligned}
\bar{D} U^{n}+q_{n}(A U) & =f^{n} \quad \text { for } n \geq 2, \\
\bar{\partial} U^{1}+k^{\alpha} \beta_{0}\left(\frac{2}{3} A U^{1}+\frac{1}{3} A U^{0}\right) & =\frac{2}{3} f^{1}+\frac{1}{3} f^{0}, \\
U^{0} & =u_{0} .
\end{aligned}
$$

The particular choice of $U^{1}$ is motivated by the fact that it will yield the desired error estimates. We note that the equation for $U^{1}$ may be written $\bar{D} U^{1}+q_{1}(A U)=$ $f^{1}+\frac{1}{2} f^{0}$, if we set $U^{-1}=u_{0}$. At each time step $t_{n}$ with $n \geq 2$, this scheme requires the solution of the equation

$$
\begin{aligned}
\left(\frac{3}{2} I+k^{1+\alpha} \beta_{0} A\right) U^{n}= & 2 U^{n-1}-\frac{1}{2} U^{n-2} \\
& -k^{1+\alpha} \sum_{j=1}^{n-1} \beta_{n-j} A U^{j}-\frac{1}{2} k^{1+\alpha} \beta_{n-1} A u_{0}+k f^{n},
\end{aligned}
$$

and a linear system with the same operator has to be solved for $U^{1}$.

For the scheme (1.18) we first derive stability and smooth data error estimates for the inhomogeneous equation with vanishing initial data, and then present our main result, the nonsmooth data estimate for the homogeneous equation,

$$
\left\|U^{n}-u\left(t_{n}\right)\right\| \leq C k^{2} t_{n}^{-2}\left\|u_{0}\right\| \text { for } n \geq 1,
$$

with $C=C_{\alpha}$, and a second-order estimate in terms of the data also for the inhomogeneous equation. As with the backward Euler method, this may be combined with (1.9) to obtain a nonsmooth data estimate in the case of an elliptic partial differential operator $A$, with $C=C_{\alpha}$,

$$
\left\|U^{n}-u\left(t_{n}\right)\right\| \leq C\left(k^{2} t_{n}^{-2}+h^{2} t_{n}^{-\alpha-1}\right)\left\|u_{0}\right\| \quad \text { for } n \geq 1, \quad \text { when } U^{0}=P_{h} u_{0} .
$$

We add a remark concerning more general kernels $\beta(t)$ : Our nonsmooth data estimates (and their proofs) remain valid on finite time intervals for kernels $\beta(t)$ such that $\hat{\beta}$ is analytic with $\left|\hat{\beta}^{(k)}(z)\right| \leq C|z|^{-\alpha-k}, k=0,1$, in a sector $|\arg z| \leq \theta$ with $\theta>\frac{1}{2} \pi$. The first- and second-order convolution quadratures are then positive definite in the sense of (1.11) provided that $\Re \hat{\beta}(z)>0$ for $\Re z>0$, i.e., whenever the kernel $\beta$ itself is positive definite.

The semidiscrete, backward Euler, and second-order backward difference methods are treated in $\S \S 2,3$, and 4 below. 


\section{The SPATIALly SEMidiscRete PROBlEM}

We shall now consider the semidiscrete problem (1.7) and show the nonsmooth data error estimate (1.9). We begin with the homogeneous equation.

Theorem 2.1. Let $\beta$ be defined by (1.4) and let $f=0$. Then we have for the solutions of (1.1) and (1.7), with $u_{0 h}=P_{h} u_{0}$,

$$
\left\|u_{h}(t)-u(t)\right\| \leq C h^{2} t^{-\alpha-1}\left\|u_{0}\right\| \quad \text { for } t>0 .
$$

Proof. Introducing the Laplace transform of the solution,

$$
\hat{u}(x, z)=\int_{0}^{\infty} u(x, t) e^{-z t} d t
$$

and recalling that $\left(u_{t}\right)^{\wedge}=-u_{0}+z \hat{u}$, we obtain from the homogeneous version of (1.1) that $z \hat{u}+\hat{\beta} A \hat{u}=u_{0}$, and hence, since $\hat{\beta}(z)=z^{-\alpha}$,

$$
\hat{u}(z)=\left(z I+z^{-\alpha} A\right)^{-1} u_{0}=\hat{E}(z) u_{0}, \quad \text { where } \hat{E}(z)=z^{\alpha}\left(z^{1+\alpha} I+A\right)^{-1} .
$$

The solution of (1.1) may therefore be obtained by the inverse Laplace transform, with integration along a line parallel to and to the right of the imaginary axis. Let now $\theta<\pi$ be a fixed number such that $\pi / 2<\theta<\pi /(1+\alpha)$. Then $\hat{E}(z)$ is analytic and satisfies $|\hat{E}(z)| \leq M /|z|$ in $\Sigma_{\theta} \cup\{0\}$, and the path of integration may therefore be deformed into the curve $\Gamma=\Gamma_{\theta}:=\{z:|\arg z|=\theta\}$ (with $\Im z$ running from $-\infty$ to $\infty$ ), so that

$$
u(t)=\frac{1}{2 \pi i} \int_{\Gamma} e^{t z} \hat{E}(z) u_{0} d z=E(t) u_{0} .
$$

This definition of $\Gamma$ will be used throughout the paper but it will be understood in what follows that when the integrand is singular at $z=0$ and not integrable along $\Gamma$, this curve will be interpreted as going around the origin to the right. In our error estimates below the integrand will consist of a difference in which the nonintegrable singularities cancel, and thus such a perturbation is then not needed.

Defining the positive definite operator $A_{h}$ on $S_{h}$ by $\left(A_{h} \psi, \chi\right)=A(\psi, \chi) \forall \psi, \chi \in$ $S_{h}$, and noting that (1.6) holds with $A$ replaced by $A_{h}$ because the minimal eigenvalue of $A_{h}$ is bounded below by that of $A$ since $S_{h}$ is a subspace of $H=L_{2}(\Omega)$, we have similarly, with $u_{0 h}=P_{h} u_{0}$,

$u_{h}(t)=\frac{1}{2 \pi i} \int_{\Gamma} e^{t z} \hat{E}_{h}(z) P_{h} u_{0} d z=E_{h}(t) P_{h} u_{0}, \quad$ where $\hat{E}_{h}(z)=z^{\alpha}\left(z^{1+\alpha} I+A_{h}\right)^{-1}$.

Introducing the error operator $F_{h}(t)=E_{h}(t) P_{h}-E(t)$, we shall show

$$
\left\|\hat{F}_{h}(z)\right\| \leq C h^{2}|z|^{\alpha} \quad \text { for } z \in \Gamma, \quad \text { where } \hat{F}_{h}(z)=\hat{E}_{h}(z) P_{h}-\hat{E}(z),
$$

which implies our claim since $u_{h}(t)-u(t)=F_{h}(t) u_{0}$ and, with $c:=\sin (\theta-\pi / 2)$,

$$
\left\|F_{h}(t)\right\|=\left\|\frac{1}{2 \pi i} \int_{\Gamma} e^{t z} \hat{F}_{h}(z) d z\right\| \leq C h^{2} \int_{\Gamma}|z|^{\alpha} e^{-c t|z|}|d z|=C h^{2} t^{-\alpha-1} .
$$

To prove (2.2), we note that since $A(z I+A)^{-1}=I-z(z I+A)^{-1}$, (1.6) shows

$$
\left\|A(z I+A)^{-1}\right\| \leq M+1 \quad \text { for }|\arg z| \leq \omega \quad(\omega<\pi),
$$


and that (2.4) also holds with $A$ replaced by $A_{h}$. We now write, with $w=z^{1+\alpha}$ for $z \in \Gamma$

$$
\begin{aligned}
\left(w I+A_{h}\right)^{-1} P_{h} & -(w I+A)^{-1}=P_{h}\left(\left(w I+A_{h}\right)^{-1}-(w I+A)^{-1}\right) P_{h} \\
- & \left(I-P_{h}\right)(w I+A)^{-1} P_{h}-(w I+A)^{-1}\left(I-P_{h}\right)=I+I I+I I I .
\end{aligned}
$$

Here, using (2.4), we have

$$
\|I I I\| \leq C\left\|A^{-1}\left(I-P_{h}\right)\right\| \leq C h^{2},
$$

where the latter inequality follows from

$$
\left(A^{-1}\left(I-P_{h}\right) v, \varphi\right)=\left(v,\left(I-P_{h}\right) A^{-1} \varphi\right) \leq\|v\| C h^{2}\left\|A^{-1} \varphi\right\|_{2} \leq C h^{2}\|v\|\|\varphi\| .
$$

Since $I I=I I I^{*} P_{h},\|I I\|$ is bounded in the same way. Also,

$$
I=\left(w I+A_{h}\right)^{-1} A_{h}\left(A_{h}^{-1} P_{h}-P_{h} A^{-1}\right) A(w I+A)^{-1} P_{h},
$$

and hence, using (2.4) and its analogue for $A_{h}$, we get

$$
\|I\| \leq C\left\|A_{h}^{-1} P_{h}-A^{-1}\right\|+\left\|\left(I-P_{h}\right) A^{-1}\right\| \leq C h^{2},
$$

where in the last step we have used the standard $L_{2}$ error estimate for the elliptic problem. Together, these estimates show (2.1) and thus complete the proof.

We now turn to the inhomogeneous equation with vanishing initial data and note that, from the Laplace transforms of the solutions of (1.1) and (1.7),

$$
u_{h}(t)-u(t)=\int_{0}^{t} F_{h}(t-s) f(s) d s .
$$

Hence, if $f(s)=0$ near $s=t$, we have by $(2.3)$

$$
\left\|u_{h}(t)-u(t)\right\| \leq C h^{2} \int_{0}^{t}(t-s)^{-\alpha-1}\|f(s)\| d s,
$$

without any regularity assumptions on $f$. In order to demonstrate an estimate valid also for $f(t) \neq 0$ we now state the following:

Theorem 2.2. Let $\beta$ be defined by (1.4) and let $u_{0}=0$. Then we have, for the solutions of (1.7) and (1.1),

$$
\left\|u_{h}(t)-u(t)\right\| \leq C h^{2}\left(t^{-\alpha}\|f(0)\|+\int_{0}^{t}(t-s)^{-\alpha}\left\|f_{t}(s)\right\| d s\right) .
$$

Proof. Setting $J_{h}(t)=\int_{0}^{t} F_{h}(s) d s$ we have, by integration by parts in (2.5),

$$
u_{h}(t)-u(t)=J_{h}(t) f(0)+\int_{0}^{t} J_{h}(t-s) f_{t}(s) d s
$$

Since the Laplace transform $\hat{J}_{h}(z)$ of $J_{h}(t)$ is $z^{-1} \hat{F}_{h}(z)$, our result follows, using (2.2), from

$$
\left\|J_{h}(t)\right\|=\left\|\frac{1}{2 \pi i} \int_{\Gamma} e^{t z} \hat{J}_{h}(z) d z\right\| \leq C h^{2} \int_{\Gamma}|z|^{\alpha-1} e^{-c t|z|}|d z|=C h^{2} t^{-\alpha} .
$$

Together, Theorems 2.1 and 2.2 show the estimate (1.9) by linearity. 


\section{THE BACKWARD EUlER METHOD}

We now consider the backward Euler time-stepping method (1.10), with $q_{n}(\varphi)$ defined by (1.15), without the term corresponding to $j=0$. The main results in this section are the nonsmooth data estimate (1.14) for the homogeneous equation, and a related estimate for the inhomogeneous equation. However, we begin by showing that the scheme studied has the properties that makes it fit into the framework of [3]. In particular, we show the appropriate smooth data estimate for the inhomogeneous equation.

We shall use the notational convention $\tilde{\omega}(\zeta)=\sum_{j=0}^{\infty} \omega_{j} \zeta^{j}$ for the discrete Laplace transform or generating function of a sequence $\left\{\omega_{j}\right\}$. Thus, in the present section the quadrature formula is

$$
q_{n}(\varphi)=k^{\alpha} \sum_{j=1}^{n} \beta_{n-j} \varphi^{j}, \quad \text { with } \tilde{\beta}(\zeta)=\sum_{j=0}^{\infty} \beta_{j} \zeta^{j}=(1-\zeta)^{-\alpha} .
$$

We begin by showing the positivity property for $q_{n}$.

Lemma 3.1. The quadrature formula $q_{n}$ in (3.1) is positive definite in the sense of (1.11), and hence the solution of (1.10) satisfies the stability estimate (1.12).

Proof. We have

$$
Q_{N}(\varphi)=k^{1+\alpha} \sum_{n=1}^{N} \sum_{j=1}^{n} \beta_{n-j} \varphi^{j} \varphi^{n}
$$

Because $\varphi^{0}$ does not appear in this formula, we can choose $\varphi^{0}:=0$ in the definition of $\tilde{\varphi}$. We want to express $Q_{N}(\varphi)$ as an integral of $\tilde{\beta}|\tilde{\varphi}|^{2}$ around the unit circle, but since the radius of convergence of the power series for $\tilde{\beta}$ is 1 , some caution is needed. We therefore write

$$
Q_{N}(\varphi)=\lim _{r \rightarrow 1^{-}} Q_{N, r}(\varphi), \quad \text { with } Q_{N, r}(\varphi):=k^{1+\alpha} \sum_{n=1}^{N} \sum_{j=1}^{n} r^{n-j} \beta_{n-j} \varphi^{j} \varphi^{n},
$$

and for $0<r<1$ obtain by a simple calculation

$$
Q_{N, r}(\varphi)=k^{1+\alpha} \frac{1}{2 \pi} \int_{0}^{2 \pi} \tilde{\beta}\left(r e^{i \theta}\right)\left|\tilde{\varphi}\left(e^{i \theta}\right)\right|^{2} d \theta .
$$

Now $\Re(1-\zeta)>0$ when $|\zeta|<1$, and hence $\Re \tilde{\beta}(\zeta)=\Re(1-\zeta)^{-\alpha}>0$ for $0<\alpha<1$. Since $Q_{N, r}(\varphi)$ is real-valued, we conclude that $Q_{N, r}(\varphi) \geq 0$ for all $\varphi \in R^{N}$. Letting $r \rightarrow 1$, we conclude that $Q_{N}(\varphi) \geq 0$ for all $\varphi \in R^{N}$.

We also need estimates for the quadrature errors $\varepsilon_{n}(\varphi)$ and $\mathcal{E}_{n}(\varphi)$ defined earlier. We note that the global error is $O(k)$, uniformly for small $t$, even though the local error exhibits a weakly singular behavior.

Lemma 3.2. We have, for $n \geq 0$,

$$
\left\|\varepsilon_{n}(\varphi)\right\| \leq C k\left\{t_{n}^{\alpha-1}\|\varphi(0)\|+\int_{0}^{t_{n}}\left(t_{n+1}-s\right)^{\alpha-1}\left\|\varphi_{t}(s)\right\| d s\right\}
$$

and, consequently, for $t_{n} \leq T$,

$$
\mathcal{E}_{n}(\varphi) \leq C_{T} k\left\{\|\varphi(0)\|+\int_{0}^{t_{n}}\left\|\varphi_{t}\right\| d s\right\} .
$$


Proof (cf. also [2]). We define

$$
q(t ; \varphi)=k^{\alpha} \sum_{0 \leq t_{j} \leq t} \beta_{j} \varphi\left(t-t_{j}\right)=\left(\bar{\beta}_{k} * \varphi\right)(t) \quad \text { for } t \geq 0,
$$

where $\bar{\beta}_{k}=k^{\alpha} \sum_{j=0}^{\infty} \beta_{j} \delta_{t_{j}}$, with $\delta_{\tau}$ the delta function concentrated at $\tau$, and note that $q(t ; \varphi)$ is continuous from the right at $t_{n}$ and that $q_{n}(\varphi)=\lim _{t \rightarrow t_{n}^{-}} q(t ; \varphi)$ for $\varphi$ continuous. We further define

$$
\varepsilon(t ; \varphi)=q(t ; \varphi)-(\beta * \varphi)(t)=\left(\gamma_{k} * \varphi\right)(t) \text { for } t \geq 0, \quad \gamma_{k}=\bar{\beta}_{k}-\beta,
$$

so that $\varepsilon_{n}(\varphi)=\lim _{t \rightarrow t_{n}^{-}} \varepsilon(t ; \varphi)$. Since $\varphi(t)=1 \cdot \varphi(0)+\left(1 * \varphi_{t}\right)(t)$, we have (note that $\left.\gamma_{k} *\left(1 * \varphi_{t}\right)=\left(\gamma_{k} * 1\right) * \varphi_{t}\right)$

$$
\varepsilon(\cdot ; \varphi)=\left(\gamma_{k} * 1\right) \varphi(0)+\left(\gamma_{k} * 1\right) * \varphi_{t}=\varepsilon(\cdot ; 1) \varphi(0)+\varepsilon(\cdot ; 1) * \varphi_{t} .
$$

To show (3.3), it is therefore enough to show

$$
|\varepsilon(t ; 1)| \leq C k(t+k)^{\alpha-1} \quad \text { for } t \geq 0 .
$$

For $t \in[0, k)$ this follows at once by $\varepsilon(t ; 1)=k^{\alpha} \beta_{0}-t^{\alpha} / \Gamma(\alpha+1)=O\left(k^{\alpha}\right)$, and it thus suffices to consider $t \geq k$. For $t \in\left[t_{n-1}, t_{n}\right), n>1$, we have $q(t ; 1)=k^{\alpha} \sum_{j=0}^{n-1} \beta_{j}$, and

$$
\sum_{j=0}^{n-1} \beta_{j}=\sum_{j=0}^{n-1}(-1)^{j}\left(\begin{array}{c}
-\alpha \\
j
\end{array}\right)=(-1)^{n-1}\left(\begin{array}{c}
-\alpha-1 \\
n-1
\end{array}\right)=\frac{n^{\alpha}}{\Gamma(\alpha+1)}+O\left(n^{\alpha-1}\right),
$$

where in the last step we have used Stirling's formula. Hence,

$$
\varepsilon(t ; 1)=q(t ; 1)-(\beta * 1)(t)=\left(t_{n}^{\alpha}-t^{\alpha}\right) / \Gamma(\alpha+1)+k^{\alpha} O\left(n^{\alpha-1}\right)=k O\left(t_{n}^{\alpha-1}\right),
$$

which completes the proof of (3.7) and thus of the lemma.

For later reference we give an alternative proof of (3.7) which also shows that Lemma 3.2 remains valid, more generally, for $\beta$ with $\hat{\beta}$ analytic and $\left|\hat{\beta}^{(k)}(z)\right| \leq$ $C|z|^{-\alpha-k}$ for $k=0,1$, in $\Sigma_{\theta}$ with $\theta>\pi / 2$. The quadrature formula is thus defined as above, now with $k^{\alpha} \beta_{j}$ the coefficient of $\zeta^{j}$ in the expansion of the analytic function $\tilde{\beta}(\zeta)=\hat{\beta}((1-\zeta) / k)$ around the origin. As before, we may restrict the discussion to $t>k$.

We have by the Laplace inversion formula, with $\Gamma$ as earlier (recall that in this case $\Gamma$ is interpreted as passing to the right of the origin),

$$
(\beta * 1)(t)=\frac{1}{2 \pi i} \int_{\Gamma} e^{z t} \hat{\kappa}(z) d z, \quad \text { where } \hat{\kappa}(z)=\hat{\beta}(z) / z .
$$

We shall prove that $q(t ; 1)=(\beta * 1)\left(t_{n-1}\right)+k O\left(t^{\alpha-1}\right)$ for $t \in\left[t_{n-1}, t_{n}\right), n>1$, which shows (3.7) since, by (3.8),

$$
\left|(\beta * 1)(t)-(\beta * 1)\left(t_{n-1}\right)\right| \leq C k \int_{\Gamma} e^{-c|z| t}|z|^{-\alpha}|d z| \leq C k t^{\alpha-1} .
$$

We have, for small $\rho$,

$$
k^{\alpha} \beta_{n}=\frac{1}{2 \pi i} \int_{|\zeta|=\rho} \zeta^{-n-1} \hat{\beta}\left(\frac{1-\zeta}{k}\right) d \zeta
$$


and hence, since $\sum_{j=0}^{n-1} \zeta^{-j-1}=\left(\zeta^{-n}-1\right) /(1-\zeta)$,

$$
q(t ; 1)=k^{\alpha} \sum_{j=0}^{n-1} \beta_{j}=\frac{k^{-1}}{2 \pi i} \int_{|\zeta|=\rho} \zeta^{-n} \hat{\kappa}\left(\frac{1-\zeta}{k}\right) d \zeta,
$$

where we have used the fact that $\hat{\beta}((1-\zeta) / k) /(1-\zeta)$ is analytic for small $\zeta$, and thus does not contribute to the integral. We now change variables $\zeta=e^{-z k}$ and deform the resulting contour $\left\{z=k^{-1} \log (1 / \rho)+i y:|y| \leq \pi / k\right\}$ to $\Gamma_{k}=\{z \in \Gamma$ : $|\Im z| \leq \pi / k\}$ (note that the contributions from the line segments on $\Im z= \pm \pi / k$ cancel by periodicity), and obtain

$$
q(t ; 1)=\frac{1}{2 \pi i} \int_{\Gamma_{k}} e^{z t_{n-1}} \hat{\kappa}\left(\frac{1-e^{-z k}}{k}\right) d z, \quad t \in\left[t_{n-1}, t_{n}\right), n>1 .
$$

Upon forming the difference of (3.10) and (3.8) (with $t=t_{n-1}$ ), the desired bound (3.7) now follows by elementary estimates: we have

$$
\left|\int_{\Gamma \backslash \Gamma_{k}} e^{z t_{n-1}} \hat{\kappa}(z) d z\right| \leq C \int_{\Gamma \backslash \Gamma_{k}} e^{-c|z| t}|z|^{-\alpha-1}|d z| \leq C k t^{\alpha-1} .
$$

Since $\left|\hat{\kappa}^{\prime}(z)\right| \leq C|z|^{-\alpha-2}$ on $\Gamma_{k}$, we obtain, with $z_{k}=\left(1-e^{-z k}\right) / k=z+O\left(k z^{2}\right)$,

$$
\left|\hat{\kappa}\left(z_{k}\right)-\hat{\kappa}(z)\right| \leq C|z|^{-\alpha-2}\left|z_{k}-z\right| \leq C k|z|^{-\alpha}, \quad z \in \Gamma_{k},
$$

and consequently

$$
\left|\int_{\Gamma_{k}} e^{z t_{n-1}}\left(\hat{\kappa}\left(z_{k}\right)-\hat{\kappa}(z)\right) d z\right| \leq C k \int_{\Gamma_{k}} e^{-c|z| t}|z|^{-\alpha}|d z| \leq C k t^{\alpha-1} .
$$

Combining these estimates proves (3.7) and hence the lemma.

We remark that (3.7) and hence (3.3) remain valid even for $\alpha \geq 1$, which is easily seen by using a contour which goes around the origin to the right, e.g., along $|z|=1 / t$. In the proof of Theorem 3.3 we shall have a use for this observation in the case $\alpha=1$.

As a result of Lemmas 3.1 and 3.2, together with (1.13), we may now conclude:

Theorem 3.1. With $q_{n}$ as in (3.1) we have, for the solutions of (1.10) and (1.1),

$$
\left\|U^{n}-u\left(t_{n}\right)\right\| \leq C_{T} k\left\{\left\|A u_{0}\right\|+\int_{0}^{t_{n}}\left(\left\|u_{t t}\right\|+\left\|A u_{t}\right\|\right) d t\right\} \quad \text { for } t_{n} \leq T .
$$

We are now ready to show our nonsmooth data error estimate for the homogeneous equation.

Theorem 3.2. If $f \equiv 0$ and $q_{n}$ is given by (3.1), we have, for the solutions of (1.10) and (1.1),

$$
\left\|U^{n}-u\left(t_{n}\right)\right\| \leq C k t_{n}^{-1}\left\|u_{0}\right\| \quad \text { for } n>0 .
$$

Proof. It turns out to be convenient for the analysis to write $u=u_{0}+v$, where $v$ thus satisfies

$$
v_{t}+\beta * A v=-(\beta * 1) A u_{0} \quad \text { for } t>0, \quad \text { with } v(0)=0 .
$$

Taking Laplace transforms, we obtain

$$
z \hat{v}(z)+\hat{\beta}(z) A \hat{v}(z)=-\hat{\beta}(z) \hat{1}(z) A u_{0},
$$

with $\hat{\beta}(z)=z^{-\alpha}$ and $\hat{1}(z)=z^{-1}$, or

$$
\hat{v}(z)=z^{-1} \hat{B}(z) u_{0}, \quad \text { where } \hat{B}(z)=-A\left(z^{1+\alpha} I+A\right)^{-1} \text {. }
$$


Thus, by applying the Laplace inversion formula, since $\hat{B}(z)$ is analytic in $\Sigma_{\theta}$, we can write

$$
v(t)=\frac{1}{2 \pi i} \int_{\Gamma} e^{t z} \hat{K}(z) d z u_{0}, \quad \hat{K}(z)=z^{-1} \hat{B}(z) .
$$

We now turn to the approximate equation (1.10) with $f \equiv 0$ and write, analogously to above, $U^{n}=u_{0}+V^{n}$, and thus obtain for our discrete problem

$$
\bar{\partial} V^{n}+q_{n}(A V)=-q_{n}(1) A u_{0} \quad \text { for } n \geq 1, \quad \text { with } V^{0}=0 .
$$

Multiplying by $\zeta^{n}$ and summing from 1 to $\infty$, we obtain for the generating function, $\tilde{V}(\zeta)=\sum_{n=0}^{\infty} V^{n} \zeta^{n}$

$$
\frac{1-\zeta}{k} \tilde{V}(\zeta)+\sum_{n=1}^{\infty} q_{n}(A V) \zeta^{n}=-\sum_{n=1}^{\infty} q_{n}(1) \zeta^{n} A u_{0} .
$$

Since $V^{0}=0$, we have $q_{n}(A V)=q_{n}^{0}(A V)$, and hence the first sum equals

$$
\sum_{n=1}^{\infty} q_{n}(A V) \zeta^{n}=\sum_{n=0}^{\infty} \sum_{j=0}^{n} k^{\alpha} \beta_{n-j} \zeta^{n-j} \cdot A V^{j} \zeta^{j}=\hat{\beta}\left(\frac{1-\zeta}{k}\right) A \tilde{V}(\zeta)
$$

for the right-hand side we have similarly, with $1_{k}:=(0,1,1,1, \ldots)$, and hence $\tilde{1}_{k}(\zeta)=\zeta /(1-\zeta)$,

$$
-\sum_{n=1}^{\infty} q_{n}(1) \zeta^{n} A u_{0}=-\tilde{1}_{k}(\zeta) \hat{\beta}\left(\frac{1-\zeta}{k}\right) A u_{0} .
$$

We therefore obtain

$$
\tilde{V}(\zeta)=\tilde{1}_{k}(\zeta) \hat{B}\left(\frac{1-\zeta}{k}\right) u_{0}
$$

This expression is analytic at $\zeta=0$, so that $\tilde{V}(\zeta)=\sum_{n=0}^{\infty} V^{n} \zeta^{n}$ has a positive radius of convergence, and hence, for $\rho$ sufficiently small,

$$
V^{n}=\frac{1}{2 \pi i} \int_{|\zeta|=\rho} \zeta^{-n}(1-\zeta)^{-1} \hat{B}\left(\frac{1-\zeta}{k}\right) d \zeta u_{0}=\frac{k^{-1}}{2 \pi i} \int_{|\zeta|=\rho} \zeta^{-n} \hat{K}\left(\frac{1-\zeta}{k}\right) d \zeta u_{0} .
$$

This formula for $V^{n}$ is of the same form as (3.9), with $\hat{K}(z) u_{0}$ instead of $\hat{\kappa}(z)$, and the representation (3.11) of $v\left(t_{n}\right)$ corresponds similarly to (3.8). Using the fact that

$$
\|\hat{K}(z)\| \leq C|z|^{-1} \text { and }\left\|\hat{K}^{\prime}(z)\right\| \leq C|z|^{-2} \text { for } z \in \Sigma_{\theta},
$$

we may therefore repeat the arguments of the proof of (3.7) with $\alpha=0$ to show

$$
\left\|V^{n}-v\left(t_{n}\right)\right\| \leq C k t_{n}^{-1}\left\|u_{0}\right\| \text { for } n \geq 1 .
$$

Since $U^{n}-u\left(t_{n}\right)=V^{n}-v\left(t_{n}\right)$, this completes the proof.

For the inhomogeneous equation with vanishing initial data we have the following $O(k)$ error bound with low regularity assumptions on data. By linearity, Theorems 3.2 and 3.3 together cover the general case.

Theorem 3.3. If $u_{0}=0$ and $q_{n}$ is given by (3.1), we have, for the solutions of (1.10) and (1.1),

$$
\left\|U^{n}-u\left(t_{n}\right)\right\| \leq C k\left(\|f(0)\|+\int_{0}^{t_{n}}\left\|f_{t}\right\| d t\right) .
$$


Proof. Taking Laplace transforms in (1.1), we have this time

$$
\hat{u}(z)=\hat{E}(z) \hat{f}(z), \quad \text { where } \hat{E}(z)=z^{\alpha}\left(z^{1+\alpha} I+A\right)^{-1},
$$

and therefore, with $E(t)$ defined as in (2.1),

$$
u(t)=(E * f)(t)=\int_{0}^{t} E(t-s) f(s) d s .
$$

Similarly to the above, from (1.10) we have, with $\tilde{f}(\zeta)=\sum_{j=1}^{\infty} f\left(t_{j}\right) \zeta^{j}$,

$$
\tilde{U}(\zeta)=\left(\frac{1-\zeta}{k} I+\hat{\beta}\left(\frac{1-\zeta}{k}\right) A\right)^{-1} \tilde{f}(\zeta)=\hat{E}\left(\frac{1-\zeta}{k}\right) \tilde{f}(\zeta)
$$

so that

$$
U^{n}=k \sum_{j=1}^{n} E_{n-j} f\left(t_{j}\right), \text { where } \hat{E}\left(\frac{1-\zeta}{k}\right)=k \sum_{j=0}^{\infty} E_{j} \zeta^{j} .
$$

Thus, $U^{n}$ has the same form as $q_{n}(f)$, with $\hat{E}(z)$ in the role of $\hat{\beta}(z)$. For the error analysis we can therefore again go through the steps of the proof of Lemma 3.2, replacing every occurrence of $k^{\alpha} \beta_{j}$ by $k E_{j}$, and $\hat{\beta}(z)$ by $\hat{E}(z)$, noting that by (1.6)

$$
\|\hat{E}(z)\| \leq C|z|^{-1} \text { and }\left\|\hat{E}^{\prime}(z)\right\| \leq C|z|^{-2} \text { for } z \in \Sigma_{\theta} .
$$

This corresponds to the case $\alpha=1$ in Lemma 3.2, and the result thus follows.

\section{A SECOND-ORDER BACKWARD DIFFERENCE METHOD}

We now turn to the second-order backward difference method. In this case the time-stepping scheme is (1.18), where, with $\delta(\zeta)=\frac{3}{2}-2 \zeta+\frac{1}{2} \zeta^{2}$,

$$
q_{n}(\varphi)=k^{\alpha}\left(\sum_{j=1}^{n} \beta_{n-j} \varphi^{j}+\frac{1}{2} \beta_{n-1} \varphi^{0}\right), \quad \text { where } \tilde{\beta}(\zeta)=\hat{\beta}(\delta(\zeta))=\delta(\zeta)^{-\alpha} .
$$

Again we begin by considering the method in the framework of [3]. It turns out that the stability of the scheme (1.18) is related to the A-stability of the backward difference approximation defined by (1.16). For completeness we include this fact, in the form of the following lemma.

Lemma 4.1. We have $\Re \delta(\zeta)=\Re\left(3-4 \zeta+\zeta^{2}\right) / 2>0$ when $|\zeta| \leq 1, \zeta \neq 1$.

Proof. With $\zeta=\xi+i \eta$ we have, for $\xi^{2}+\eta^{2} \leq 1, \xi \neq 1$,

$$
\Re \delta(\zeta)=\frac{1}{2}\left(\xi^{2}-\eta^{2}-4 \xi+3\right) \geq \xi^{2}-2 \xi+1>0 .
$$

We are now in a position to show the stability of (1.18).

Lemma 4.2. The scheme (1.18), with $q_{n}$ as in (4.1), is stable in the sense that

$$
\left\|U^{n}\right\| \leq C\left\{\left\|u_{0}\right\|+k \sum_{j=0}^{n}\left\|f^{j}\right\|\right\} \quad \text { for } n \geq 0 .
$$


Proof. By linearity it suffices to consider the cases $u_{0}=0$ and $f=0$ separately. In the case $u_{0}=0$ we shall show that $Q_{N}(\varphi) \geq 0$, where $Q_{N}(\varphi)$ is as in (3.2). The proof is analogous to that in Lemma 3.1, using this time the fact that $\Re \tilde{\beta}(\zeta)=$ $\Re \delta(\zeta)^{-\alpha} \geq 0$ when $|\zeta| \leq 1$ and $0<\alpha<1$, since $\Re \delta(\zeta) \geq 0$ by Lemma 4.1. Once this is known, the proof proceeds as in [3]; the fact that the equation at $t=t_{1}$ is different from that in [3] does not present any difficulty if we take $t_{-1}$ as the starting level, and use $U^{0}=U^{-1}=0$.

In the case $f=0, U^{n}$ solves the homogeneous equation. We then appeal to our Theorem 4.2 below which shows, using the stability of the continuous problem,

$$
\left\|U^{n}\right\| \leq\left\|U^{n}-u\left(t_{n}\right)\right\|+\left\|u\left(t_{n}\right)\right\| \leq C n^{-2}\left\|u_{0}\right\|+C\left\|u_{0}\right\| \leq C\left\|u_{0}\right\| .
$$

We now show that the quadrature rule $q_{n}$ is second-order accurate away from $t=0$, with the global error uniformly $O\left(k^{2}\right)$ for small $t$ when $\varphi(0)=0$. We remark that, as in Lemma 3.2, the result is valid for any kernel $\beta$ with $\hat{\beta}$ analytic and such that $\left|\hat{\beta}^{(k)}(z)\right| \leq M|z|^{-\alpha-k}, k=0,1$, in $\Sigma_{\theta}$, with $\theta>\pi / 2$.

Lemma 4.3. For the quadrature formula (4.1) we have for $n \geq 0$

$$
\left\|\varepsilon_{n}(\varphi)\right\| \leq C k^{2}\left\{t_{n}^{\alpha-2}\|\varphi(0)\|+t_{n}^{\alpha-1}\left\|\varphi_{t}(0)\right\|+\int_{0}^{t_{n}}\left(t_{n+1}-s\right)^{\alpha-1}\left\|\varphi_{t t}(s)\right\| d s\right\}
$$

and, consequently, if $\varphi(0)=0$,

$$
\mathcal{E}_{n}(\varphi) \leq C_{T} k^{2}\left\{\left\|\varphi_{t}(0)\right\|+\int_{0}^{t_{n}}\left\|\varphi_{t t}\right\| d s\right\} \quad \text { for } t_{n} \leq T .
$$

Proof. We begin by considering the case $\varphi(0)=0$, and write $\varphi(t)=t \cdot \varphi_{t}(0)+$ $\int_{0}^{t}(t-s) \varphi_{t t}(s) d s$, or $\varphi=\varphi_{t}(0) \tau+\tau * \varphi_{t t}$, where $\tau(t) \equiv t$. Defining $q(t ; \varphi)$ and $\varepsilon(\cdot ; \varphi)$ as in (3.4) and (3.5), we find, with $\bar{\beta}_{k}=k^{\alpha} \sum_{0}^{\infty} \beta_{j} \delta_{t_{j}}$,

$$
\varepsilon(t ; \varphi)=\left(\left(\bar{\beta}_{k}-\beta\right) * \varphi\right)(t)=\varepsilon(t ; \tau) \varphi_{t}(0)+\left(\varepsilon(\cdot ; \tau) * \varphi_{t t}\right)(t) \quad \text { for } t \geq 0 .
$$

(Note that now, for $\varphi$ continuous with $\varphi(0)=0, q(t ; \varphi)$ is continuous at $t=t_{n}$, that $\lim _{t \rightarrow t_{n}} q(t ; \varphi)=q_{n}(\varphi)$, and that $\varepsilon(t ; \varphi)$ has analogous properties.) It thus suffices to show

$$
|\varepsilon(t ; \tau)| \leq C k^{2}(t+k)^{\alpha-1} \quad \text { for } t \geq 0 .
$$

For $t \in[0, k)$, we have $\varepsilon(t ; \tau)=k^{\alpha} \beta_{0} t-(\beta * \tau)(t)=O\left(k^{\alpha+1}\right)$, and for $t \in$ $\left[t_{n-1}, t_{n}\right), n>1$,

$$
q(t ; \tau)=k^{\alpha} \sum_{j=0}^{n-1} \beta_{j}\left(t-t_{j}\right)=\left(t-t_{n}\right) q_{n-1}^{0}(1)+q_{n}(\tau), \quad \text { where } q_{n-1}^{0}(1)=\sum_{j=0}^{n-1} \beta_{j} .
$$

We shall show below that

$$
\begin{aligned}
q_{n-1}^{0}(1) & =(\beta * 1)\left(t_{n}\right)+O\left(k t_{n}^{\alpha-1}\right), \\
q_{n}(\tau) & =(\beta * \tau)\left(t_{n}\right)+O\left(k^{2} t_{n}^{\alpha-1}\right) .
\end{aligned}
$$

Since

$$
(\beta * \tau)(t)=(\beta * \tau)\left(t_{n}\right)+\left(t-t_{n}\right)(\beta * 1)\left(t_{n}\right)+\int_{t_{n}}^{t}(t-s) \beta(s) d s,
$$

and the latter integral is of order $O\left(k^{2} t_{n}^{\alpha-1}\right)$, these estimates imply (4.2). 
To show (4.4), we note that, with $\rho$ small,

$$
k^{\alpha} \beta_{j}=\frac{1}{2 \pi i} \int_{|\zeta|=\rho} \zeta^{-j-1} \hat{\beta}\left(\frac{\delta(\zeta)}{k}\right) d \zeta,
$$

and hence

$$
q_{n-1}^{0}(1)=k^{\alpha} \sum_{j=0}^{n-1} \beta_{j}=\frac{1}{2 \pi i} \int_{|\zeta|=\rho} g(\zeta) \hat{\beta}\left(\frac{\delta(\zeta)}{k}\right) d \zeta,
$$

where, using the factorization $\delta(\zeta)=\frac{1}{2}(1-\zeta)(3-\zeta)$ and setting $\mu(\zeta)=\frac{1}{2}(3-\zeta) \zeta$, one has

$$
g(\zeta)=\sum_{j=0}^{n-1} \zeta^{-j-1}=\zeta^{-1} \frac{1-\zeta^{-n}}{1-\zeta^{-1}}=\zeta^{-n-1} \frac{\mu(\zeta)}{\delta(\zeta)}-\frac{1}{1-\zeta} .
$$

Hence, since the last term is analytic near $\zeta=0$, we have, with $\hat{\kappa}(z)=\hat{\beta}(z) / z$,

$$
q_{n-1}^{0}(1)=\frac{k^{-1}}{2 \pi i} \int_{|\zeta|=\rho} \zeta^{-n-1} \mu(\zeta) \hat{\kappa}\left(\frac{\delta(\zeta)}{k}\right) d \zeta .
$$

Making the transformation $\zeta=e^{-k z}$ and setting $z_{k}=\delta\left(e^{-k z}\right) / k$, we obtain

$$
q_{n-1}^{0}(1)=\frac{1}{2 \pi i} \int_{\Gamma_{k}} e^{t_{n} z} \mu\left(e^{-k z}\right) \hat{\kappa}\left(z_{k}\right) d z
$$

again with $\Gamma_{k}=\{z \in \Gamma:|\Im z| \leq \pi / k\}$, and hence, with $\varepsilon_{n}^{0}(1)=q_{n-1}^{0}(1)-(\beta * 1)\left(t_{n}\right)$,

$$
\varepsilon_{n}^{0}(1)=\frac{1}{2 \pi i} \int_{\Gamma_{k}} e^{t_{n} z}\left(\mu\left(e^{-k z}\right) \hat{\kappa}\left(z_{k}\right)-\hat{\kappa}(z)\right) d z-\frac{1}{2 \pi i} \int_{\Gamma \backslash \Gamma_{k}} e^{t_{n} z} \hat{\kappa}(z) d z .
$$

We rewrite the first integrand as

$$
\mu\left(e^{-k z}\right) \hat{\kappa}\left(z_{k}\right)-\hat{\kappa}(z)=\left(\mu\left(e^{-k z}\right)-1\right) \hat{\kappa}\left(z_{k}\right)+\left(\hat{\kappa}\left(z_{k}\right)-\hat{\kappa}(z)\right) .
$$

Since $\mu\left(e^{-w}\right)=1+O(w)$ and $c|z| \leq\left|z_{k}\right| \leq C|z|$ on $\Gamma_{k}$, and since $|\hat{\kappa}(z)| \leq C|z|^{-\alpha-1}$, the first term is bounded by $C k|z|^{-\alpha}$. Further, since $z_{k}-z=O\left(k^{2} z^{3}\right)$ for bounded $k z$, and $\left|\hat{\kappa}^{\prime}(z)\right| \leq C|z|^{-\alpha-2}$, we obtain

$$
\left|\hat{\kappa}\left(z_{k}\right)-\hat{\kappa}(z)\right| \leq C k^{2}|z|^{-\alpha+1} \leq C k|z|^{-\alpha} \quad \text { for } z \in \Gamma_{k} .
$$

It follows that

$$
\left|\varepsilon_{n}^{0}(1)\right| \leq C k \int_{\Gamma_{k}} e^{-c t_{n}|z|}|z|^{-\alpha}|d z|+C \int_{\Gamma \backslash \Gamma_{k}} e^{-c t_{n}|z|}|z|^{-\alpha-1}|d z| \leq C k t_{n}^{\alpha-1},
$$

which is (4.4).

We now turn to (4.5) and note similarly to the above that $(\beta * \tau)^{\wedge}(z)=\kappa_{1}(z):=$ $\hat{\beta}(z) / z^{2}$, and that $\sum_{j=0}^{\infty} j \zeta^{j}=\zeta /(1-\zeta)^{2}$, and hence

$$
q_{n}(\tau)=k^{\alpha+1} \sum_{j=0}^{n-1} \beta_{j}(n-j)=\frac{k^{-1}}{2 \pi i} \int_{|\zeta|=\rho} \zeta^{-n-1} \mu_{1}(\zeta) \hat{\kappa}_{1}\left(\frac{\delta(\zeta)}{k}\right) d \zeta,
$$

where $\mu_{1}(\zeta)=\zeta(3-\zeta)^{2} / 4$. Setting once more $\zeta=e^{-k z}$, we find

$$
\varepsilon_{n}(\tau)=\frac{1}{2 \pi i} \int_{\Gamma_{k}} e^{t_{n} z}\left(\mu_{1}\left(e^{-k z}\right) \hat{\kappa}_{1}\left(z_{k}\right)-\hat{\kappa}_{1}(z)\right) d z-\frac{1}{2 \pi i} \int_{\Gamma \backslash \Gamma_{k}} e^{t_{n} z} \hat{\kappa}_{1}(z) d z .
$$

Now $\mu_{1}\left(e^{-w}\right)=1+O\left(w^{2}\right)$, and we conclude as above, using (4.6), that

$$
\left|\mu_{1}\left(e^{-k z}\right) \hat{\kappa}_{1}\left(z_{k}\right)-\hat{\kappa}_{1}(z)\right| \leq C k^{2}|z|^{-\alpha} \quad \text { for } z \in \Gamma_{k} .
$$


From this we obtain easily that $\left|\varepsilon_{n}(\tau)\right| \leq C k^{2} t_{n}^{\alpha-1}$, which is (4.5).

It remains to show the result of Lemma 4.3 when $\varphi(t) \equiv \varphi(0) \neq 0$, i.e., that $\varepsilon_{n}(1)=O\left(k^{2} t_{n}^{\alpha-2}\right)$. Setting $1_{k}=\left(0, \frac{3}{2}, 1,1, \ldots\right)$, we have now $q_{n}(1)=q_{n}^{0}\left(1_{k}\right)$ and hence

$$
\sum_{j=1}^{\infty} q_{n}(1) \zeta^{n}=\tilde{1}_{k}(\zeta) k^{\alpha} \tilde{\beta}(\zeta)=\frac{3 \zeta-\zeta^{2}}{2(1-\zeta)} \hat{\beta}\left(\frac{\delta(\zeta)}{k}\right)=k^{-1} \mu_{2}(\zeta) \kappa\left(\frac{\delta(\zeta)}{k}\right),
$$

where $\mu_{2}(\zeta)=\zeta(3-\zeta)^{2} / 4$, so that

$$
q_{n}(1)=\frac{k^{-1}}{2 \pi i} \int_{|\zeta|=\rho} \zeta^{-n-1} \mu_{2}(\zeta) \hat{\kappa}\left(\frac{\delta(\zeta)}{k}\right) d \zeta=\frac{1}{2 \pi i} \int_{\Gamma_{k}} e^{t_{n} z} \mu_{2}\left(e^{-k z}\right) \hat{\kappa}\left(z_{k}\right) d z
$$

Therefore,

$$
\varepsilon_{n}(1)=\frac{1}{2 \pi i} \int_{\Gamma_{k}} e^{t_{n} z}\left(\mu_{2}\left(e^{-k z}\right) \hat{\kappa}\left(z_{k}\right)-\hat{\kappa}(z)\right) d z-\frac{1}{2 \pi i} \int_{\Gamma \backslash \Gamma_{k}} e^{t_{n} z} \hat{\kappa}(z) d z .
$$

Now $\mu_{2}\left(e^{-w}\right)=1+O\left(w^{2}\right)$ so that, with (4.6),

$$
\left|\mu_{2}\left(e^{-k z}\right) \hat{\kappa}\left(z_{k}\right)-\hat{\kappa}(z)\right| \leq C k^{2}|z|^{-\alpha+1} \quad \text { for } z \in \Gamma_{k},
$$

and it follows that $\left|\varepsilon_{n}(1)\right| \leq C k^{2} t_{n}^{\alpha-2}$, which completes the proof.

Using the above Lemmas 4.2 and 4.3 , one easily shows the following optimalorder error estimates for the inhomogeneous equation with vanishing initial data.

Theorem 4.1. Under the appropriate regularity assumptions we have for the solutions of (1.18) with (4.1) and (1.1) with $u_{0}=0$,

$$
\left\|U^{n}-u\left(t_{n}\right)\right\| \leq C k^{2}\left\{\left\|A u_{t}(0)\right\|+\int_{0}^{t_{n}}\left(\left\|u_{t t t}\right\|+\left\|A u_{t t}\right\|\right) d t\right\} .
$$

We now turn to the homogeneous equation and prove the following nonsmooth data estimate.

Theorem 4.2. For $f=0$ we have for the solutions of (1.18) with (4.1) and (1.1)

$$
\left\|U^{n}-u\left(t_{n}\right)\right\| \leq C k^{2} t_{n}^{-2}\left\|u_{0}\right\| \quad \text { for } n \geq 1 .
$$

Proof. Writing again $U^{n}=u_{0}+V^{n}$ for $n \geq 0$, we may write our scheme, since $q_{n}(\varphi)=q_{n}^{0}(\varphi)$ when $\varphi^{0}=0$, as

$$
\bar{D} V^{n}+q_{n}^{0}(A V)=-q_{n}(1) A u_{0} \quad \text { for } n \geq 1, \quad \text { with } V^{0}=V^{-1}=0 .
$$

With the above notation, we obtain for the generating functions

or

$$
\left(\frac{\delta(\zeta)}{k} I+\hat{\beta}\left(\frac{\delta(\zeta)}{k}\right) A\right) \tilde{V}(\zeta)=-\hat{\beta}\left(\frac{\delta(\zeta)}{k}\right) \tilde{1}_{k}(\zeta) A u_{0}, \quad \text { with } \tilde{1}_{k}(\zeta)=\frac{3 \zeta-\zeta^{2}}{2(1-\zeta)},
$$

$$
\tilde{V}(\zeta)=\tilde{1}_{k}(\zeta) \hat{B}\left(\frac{\delta(\zeta)}{k}\right) u_{0}, \quad \text { where } \hat{B}(z)=-A\left(z^{1+\alpha} I+A\right)^{-1} .
$$

Hence, proceeding as in the proof of Lemma 4.3, we have, with $\hat{K}(z)=z^{-1} \hat{B}(z)$,

$$
V^{n}=\frac{k^{-1}}{2 \pi i} \int_{|\zeta|=\rho} \zeta^{-n-1} \mu_{2}(\zeta) \hat{K}\left(\frac{\delta(\zeta)}{k}\right) d \zeta u_{0}=\frac{1}{2 \pi i} \int_{\Gamma_{k}} e^{t_{n} z} \mu_{2}\left(e^{-k z}\right) \hat{K}\left(z_{k}\right) d z u_{0}
$$

and hence, with $G_{k}(z)=\mu_{2}\left(e^{-k z}\right) \hat{K}\left(z_{k}\right)-\hat{K}(z)$,

$$
V^{n}-v\left(t_{n}\right)=\frac{1}{2 \pi i} \int_{\Gamma_{k}} e^{t_{n} z} G_{k}(z) d z u_{0}-\frac{1}{2 \pi i} \int_{\Gamma \backslash \Gamma_{k}} e^{t_{n} z} \hat{K}(z) d z u_{0} .
$$


Using (3.12), one shows this time $\left\|G_{k}(z)\right\| \leq C k^{2}|z|$ on $\Gamma_{k}$, and concludes

$$
\left\|V^{n}-v\left(t_{n}\right)\right\| \leq C k^{2} t_{n}^{-2}\left\|u_{0}\right\|
$$

which completes the proof.

We remark that assuming $u_{0} \in D(A)$ rather than just $u_{0} \in H$ reduces the singular behavior of the error bound at $t=0$. In fact, similarly to above one may show $\left\|A^{-1} G_{k}(z)\right\| \leq C k^{2}|z|^{-\alpha}$ on $\Gamma_{k}$, and hence

$$
\left\|V^{n}-v\left(t_{n}\right)\right\| \leq C k^{2} \int_{\Gamma}|z|^{-\alpha} e^{-c t_{n}|z|}|d z|\left\|A u_{0}\right\|=C k^{2} t_{n}^{\alpha-1}\left\|A u_{0}\right\| .
$$

In particular, this shows a uniform error bound of order $O\left(k^{1+\alpha}\right)$ for $t_{n} \geq 0$, which conforms with the estimate in [3] for second-order schemes with $\beta$ as in (1.4).

We end with an optimal-order error estimate for the inhomogeneous equation with low regularity assumptions on data.

Theorem 4.3. For $u_{0}=0$ we have, for the solutions of (1.18) with (4.1) and (1.1),

$$
\left\|U^{n}-u\left(t_{n}\right)\right\| \leq C k^{2}\left\{t_{n}^{-1}\|f(0)\|+\left\|f_{t}(0)\right\|+\int_{0}^{t_{n}}\left\|f_{t t}\right\| d t\right\}
$$

Proof. We proceed as in the proof of Theorem 3.3 and obtain, setting $U^{0}=0$,

$\tilde{U}(\zeta)=\hat{E}\left(\frac{\delta(\zeta)}{k}\right)\left\{\sum_{j=2}^{\infty} f\left(t_{n}\right) \zeta^{n}+\left(f\left(t_{1}\right)+\frac{1}{2} f(0)\right) \zeta\right\}, \quad$ where $\hat{E}(z)=z^{\alpha}\left(z^{1+\alpha} I+A\right)^{-1}$,

so that $U^{n}$ is the convolution quadrature approximation of $E * f$ defined by

$$
U^{n}=k \sum_{j=1}^{n} E_{n-j} f\left(t_{j}\right)+\frac{1}{2} k E_{n-1} f(0), \quad \text { where } \quad \hat{E}\left(\frac{\delta(\zeta)}{k}\right)=k \sum_{j=0}^{\infty} E_{j} \zeta^{j} .
$$

The result thus follows as in Lemma 4.3, with $\hat{E}(z)$ in the role of $\hat{\beta}(z)$ and $\alpha=1$.

\section{REFERENCES}

1. J.-C. López-Marcos, A difference scheme for a nonlinear partial integrodifferential equation, SIAM J. Numer. Anal. 27 (1990), 20-31. MR 91e:65160

2. C. Lubich, Convolution quadrature and discretized operational calculus. I, Numer. Math. 52 (1988), 129-145. MR 89g:65018

3. W. McLean and V. Thomée, Numerical solution of an evolution equation with a positive type memory term, J. Austral. Math. Soc. Ser. B 35 (1993), 23-70. MR 94e:65094

4. W. McLean, V. Thomée, and L.B. Wahlbin, Discretization with variable time steps of an evolution equation with a positive type memory term, Applied Mathematics Report, vol. AMR93/18, School of Mathematics, University of New South Wales.

5. O. Nevanlinna, On the numerical solutions of some Volterra equations on infinite intervals, Rev. Anal. Numér. Théorie Approximation 5 (1976), 31-57. MR 58:25043 
6. J.M. Sanz-Serna, A numerical method for a partial integro-differential equation, SIAM J. Numer. Anal. 25 (1988), 319-327. MR 89d:65113

7. V. Thomée, Galerkin finite element methods for parabolic problems, Lecture Notes in Math., vol. 1054, Springer-Verlag, Berlin and New York, 1984. MR 86k:65006

Mathematisches Institut, Universität Tübingen, Auf der Morgenstelle 10, D-72076 Tübingen, Germany

E-mail address: lubich@na.mathematik.uni-tuebingen.de

School of Mathematics, University of New South Wales, Sydney 2052, Australia

E-mail address: I.Sloan@unsw.edu.au

Department of Mathematics, Chalmers University of Technology, S-412 96 Göteborg, SWEDEN

E-mail address: thomee@math.chalmers.se 